Magnetic filter field dependence of the performance of the RF driven IPP prototype source for negative hydrogen ions

This article has been downloaded from IOPscience. Please scroll down to see the full text article.

2011 Plasma Phys. Control. Fusion 53115006

(http://iopscience.iop.org/0741-3335/53/11/115006)

View the table of contents for this issue, or go to the journal homepage for more

Download details:

IP Address: 130.183.203.10

The article was downloaded on 26/01/2012 at 11:11

Please note that terms and conditions apply. 


\title{
Magnetic filter field dependence of the performance of the RF driven IPP prototype source for negative hydrogen ions
}

\author{
P Franzen, L Schiesko, M Fröschle, D Wünderlich, U Fantz and \\ the NNBI Team \\ Max-Planck-Institut für Plasmaphysik (IPP), EURATOM Association, D-85748 Garching, \\ Germany \\ E-mail: peter.franzen@ipp.mpg.de
}

\begin{abstract}
The ITER neutral beam system requires a negative hydrogen ion beam of $48 \mathrm{~A}$ with an energy of $0.87 \mathrm{MeV}$ and a negative deuterium beam of $40 \mathrm{~A}$ with an energy of $1 \mathrm{MeV}$. The beam is extracted from a large RF driven ion source with the dimension of $1.9 \times 0.9 \mathrm{~m}^{2}$. An important role for the transport of the negative hydrogen ions to the extractor and the suppression of the co-extracted electrons is the magnetic filter field in front of the extractor. For the large ITER source the filter field will be generated by a current of up to $4 \mathrm{kA}$ flowing through the first grid of the extractor. The extrapolation of the results obtained with the small IPP RF prototype source, where the filter field has a different 3D structure as it is generated by permanent magnets, is not straightforward. Furthermore, the filter field is by far not optimized due to the technical constraints of the RF source. Therefore, a frame that surrounds the ion sources and hosts permanent magnets was constructed for a fast and flexible change of the filter field. First results in hydrogen show that a minimum field of $3 \mathrm{mT}$ in front of the extractor is needed for a sufficiently large number of extracted negative hydrogen ions, whereas sufficient co-extracted electron suppression is achieved by a source integrated magnetic field of more than $1.0 \mathrm{mTm}$.
\end{abstract}

(Some figures in this article are in colour only in the electronic version)

\section{Introduction}

One of the key components of a neutral beam injection system is the ion source. The ITER neutral beam system [1-3] consists of two $1 \mathrm{MeV} / 17 \mathrm{MW}$ heating beams (HNB) and a $100 \mathrm{kV} / 3 \mathrm{MW}$ diagnostic beam (DNB). In order to achieve the required accelerated ion currents (48 $\mathrm{A}$ at $870 \mathrm{keV}$ in hydrogen, $40 \mathrm{~A}$ at $1 \mathrm{MeV}$ in deuterium), the source has to deliver negative 
ion currents of up to $70 \mathrm{~A}$ due to the stripping losses in the accelerator. (The required extracted current ratings are $70 \mathrm{~A} \mathrm{H}^{-}$for the diagnostic neutral beam injector, $68 \mathrm{~A} \mathrm{H}^{-}$and $59 \mathrm{~A} \mathrm{D}^{-}$ for the heating neutral beam injectors $[1,2]$ ). As the current densities of negative hydrogen or deuterium ions that have been achieved up to now are in the range of several $100 \mathrm{~A} \mathrm{~m}^{-2}$ only, the ion source area has to be rather large with dimensions of $1.9 \times 0.9 \mathrm{~m}^{2}$ and an extraction area of $0.2 \mathrm{~m}^{2}$.

The IPP RF driven cesiated negative hydrogen ion source is since 2007 the ITER reference source [3]; the source is principally maintenance free-only the Cs supply has to be exchanged regularly. At IPP, the ITER requirements have been fulfilled with a small prototype source and for short pulses (4s) [4-7], i.e. accelerated current densities of more than $200 \mathrm{~A} \mathrm{~m}^{-2} \mathrm{D}^{-}$and $350 \mathrm{~A} \mathrm{~m}^{-2} \mathrm{H}^{-}$with a ratio of the co-extracted electrons to ions of less than one at the required low source pressure $(0.3 \mathrm{~Pa})$ have been achieved.

An important role for the negative hydrogen ion transport and for the suppression of the coextracted electron current in cesiated sources, i.e. in sources operating with surface production of the negative ions, is the magnetic filter field (see [4, 8] for the RF driven negative ion source, and [9-14] for the arc driven sources). The magnetic filter field separates the plasma production zone (in the RF source the so-called driver where the RF is coupled in) from the extraction zone near the first grid of the acceleration system, the plasma grid. As a consequence, an extended rather complex boundary layer is formed near the plasma grid where all the processes take place leading to negative ion production and extraction as well for electron suppression [15].

The understanding of the boundary layer including the magnetic filter field in a negative ion source has strongly improved during the last decade, both by experiments and modelling (see, for example, [16-20]). To the present knowledge, the magnetic filter field in the source has two functions. The first is to cool down the electrons, for what the axial integral of the magnetic filter field or gradients might be important parameters. Measurements showed that the electron temperature in the IPP prototype RF source is reduced by sufficiently strong fields from about $10 \mathrm{eV}$ in the driver to less than $2 \mathrm{eV}$ in the boundary layer [21,22]. This low electron temperature minimizes the collissional losses of negative hydrogen ions. As the binding energy of the electron is rather low $(0.75 \mathrm{eV})$, the mean free path of a negative hydrogen ion in the main source plasma is only in the range of a few $\mathrm{cm}$ [23]. Hence, only negative ions created near the plasma grid apertures can be extracted, here the mean free path is considerably larger (several tens of $\mathrm{cm}$ ) due to the lower electron temperature and density. As these 'surface' created negative hydrogen ions are firstly accelerated into the source by the sheath potential, they have to be bent back to the plasma grid apertures for extraction. Apart from charge exchange collisions, this is done-as the second function-by the magnetic filter field; calculations showed that some magnetic field near the plasma grid is necessary for an extraction probability of the negative ions of 10-20\% [16].

The filter field causes drifts in the plasma-probably by a $E \times B$ drift-leading to a asymmetry mainly of the electron density in front of the plasma grid $[6,12]$. How this asymmetry affects the homogeneity of the negative hydrogen ion production at the plasma grid and the final beam homogeneity is still an open issue; first results [15] for the IPP prototype RF source showed some correlations of the beam homogeneity with the homogeneity of the plasma in front of the plasma grid, but recent results with an improved database indicate almost no correlation [24].

The magnetic filter field in the RF driven cesiated negative hydrogen ion source is by far not optimized with respect to the strength, position, gradients, etc for maximum negative ion yield and electron suppression. The ITER requirements have been obtained in the small IPP prototype source $\left(0.5 \times 0.3 \mathrm{~m}^{2}\right)$ with the magnetic filter field created by permanent magnets $[4,5]$. How these results can be transferred to the large ITER sources, where the field 


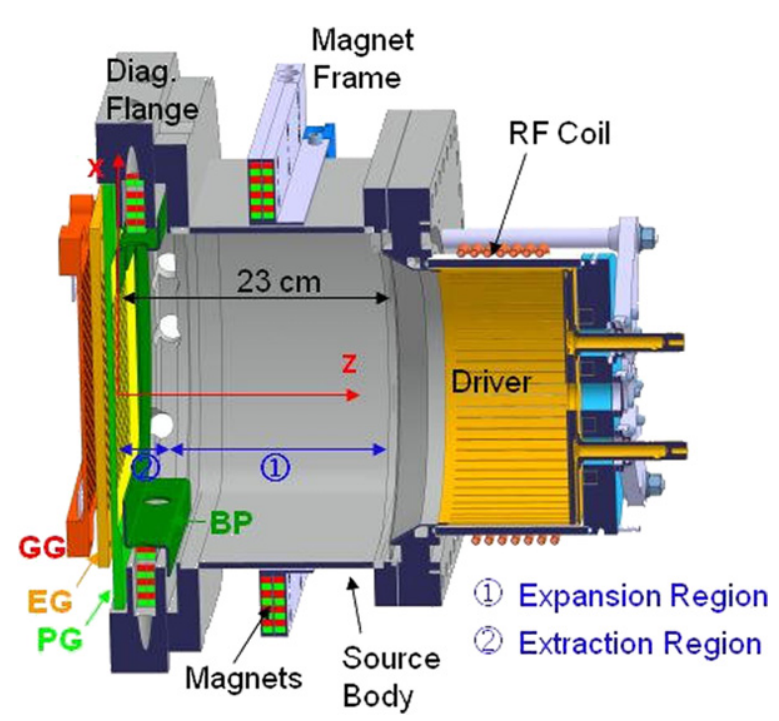

Figure 1. Horizontal section cut through the ion source and the grid system with magnets in the diagnostic flange and with the magnet frame. BP: bias plate, PG: plasma grid, EG: extraction grid, GG: grounded grid. Also indicated is the coordinate system centred in the centre of the plasma grid.

must be created by a current through the plasma grid with a different 3D field map [25, 26], is still an open issue. Hence it is important to identify the governing parameters for electron suppression and negative ion yield as input for the design of the filter field for the large ITER source.

In the IPP RF prototype source, the permanent magnets have been originally embedded internally in the so-called diagnostic flange located near the plasma grid and allowing diagnostic access to the boundary layer (see figure 1, where a horizontal section cut through the source is shown). In this setup, the magnetic filter field is at maximum in a distance of about $3 \mathrm{~cm}$ from the plasma grid. This design, however, has almost no flexibility in changing the magnetic filter field without lengthy venting procedures. The only possibility was to change the internal field by magnet rods attached from outside, but this method had the disadvantage that also the 3D structure of the field was changed [4]. Hence a new flexible magnet frame was built and installed outside at the source body of the IPP RF prototype source, allowing fast change of the filter field as well as to some extent a separation of the influence of the filter field parameters.

The experiments have been performed at the IPP test facility BATMAN [4]. Here, restrictions in the $\mathrm{HV}$ and the pumping system allow pulses up to $4 \mathrm{~s}$ only. For extraction, the so-called 'large area grid' system [27] was used with an extraction area of $63 \mathrm{~cm}^{2}$. The LAG system consists of three grids: plasma grid (PG), extraction grid (EG), which filters also the co-extracted electrons out of the beam by embedded magnets, and the grounded grid (GG). All the experimental results showed in this paper have been obtained with hydrogen.

The currents densities quoted in this paper-the current density of extracted negative ions $j_{\mathrm{ex}}$ and of co-extracted electrons $j_{\mathrm{e}}$, respectively - are derived from the electrical current flowing onto the extraction grid $\left(j_{\mathrm{e}}\right)$ and from the current flowing back from ground potential (grounded grid, scrapers, and calorimeter) to the HV power supply $\left(j_{\mathrm{ex}}\right)$, for details see [4]. Depending on the ion optics, some ions may hit the extraction grid; but we assume in the following that the extraction grid current refers totally to the co-extracted electrons. On the other hand, dedicated experiments [4] demonstrated that electron leakage out of the extraction 


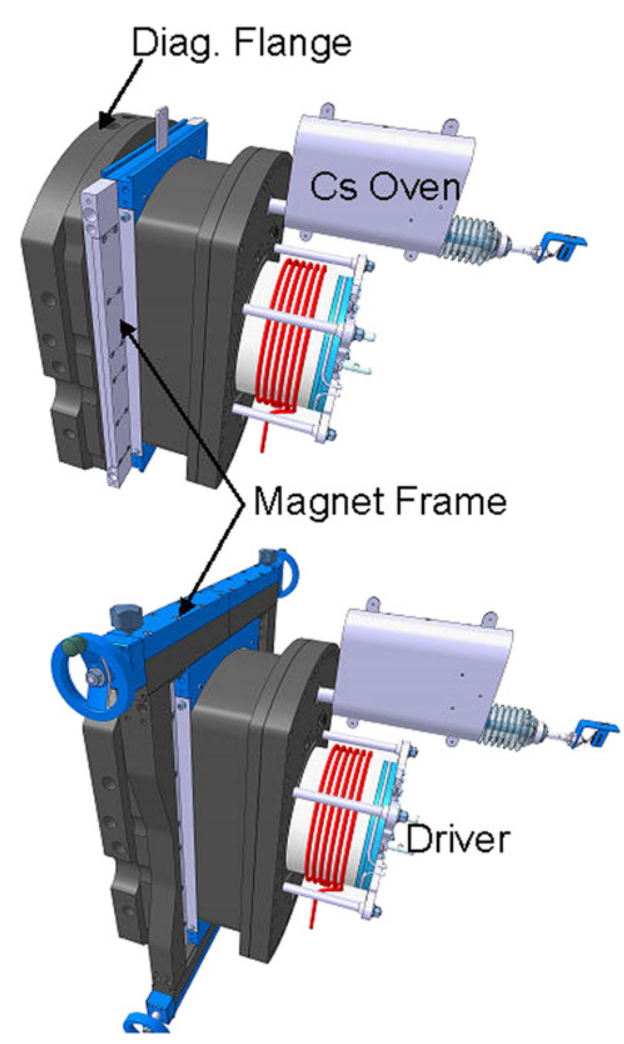

Figure 2. Top: sketch of the IPP prototype source with the magnet frame without yoke; bottom: with yoke in closed position.

grid is negligible; hence, $j_{\mathrm{ex}}$ is a measurement of the negative ions that are extracted from the ion source.

\section{Experimental setup}

Figure 1 shows a horizontal section cut through the IPP RF prototype source. The source consists of three parts: the driver, where the RF power - up to $100 \mathrm{~kW}$ at $1 \mathrm{MHz}$ frequencyis coupled in and the plasma is created, the expansion region and the extraction region in front of the plasma grid. The magnetic filter field is located in the expansion region and separates the driver from the extraction region. Caesium which is needed for the reduction of the work function of the plasma grid leading to an enhancement of the surface production rate of the negative ions is evaporated into the source by a caesium oven attached at the upper rear part of the source (see figure 2). For sufficient electron suppression, the plasma grid must be biased positively with $10-20 \mathrm{~V}$ against the source body. This plasma grid bias is supported by a so-called bias plate which is connected to the source body. It decreases the biased area by extending the source potential near to the apertures.

Figure 1 shows also the magnetic filter field setups used for the experimental results reported here: the standard configuration with internal magnets embedded in the diagnostic flange and the new external, flexible magnet frame. The design of the frame aimed to have optimum flexibility for changes of the magnetic filter field. In order not to disturb the external field, all magnets within the source have been removed. The frame can be moved continuously 


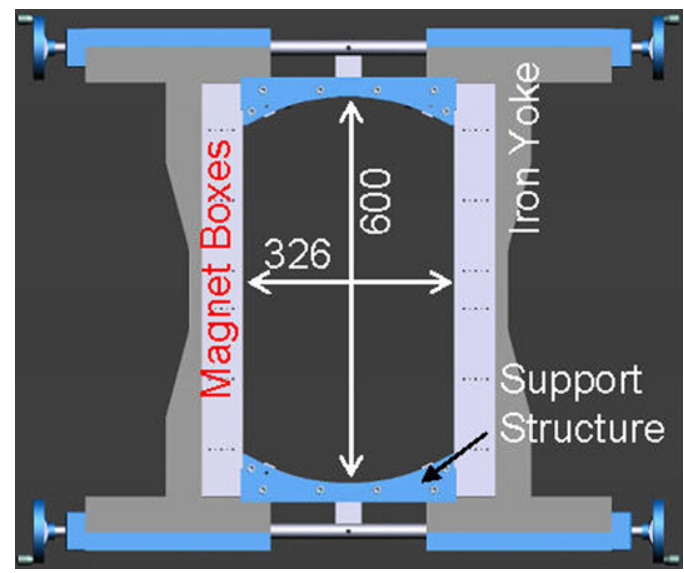

Figure 3. Magnet frame with the yoke opened. Dimensions are given in $\mathrm{mm}$.

along the source body from the diagnostic flange - the nearest position to the plasma grid - to the driver exit. Additionally, an iron yoke can be attached at the sides and on top and bottom of the frame leading to a enhancement and a larger homogeneity of the filter field in the source (see figure 2).

Figure 3 shows the details of the magnet frame; it consists mainly of three parts:

- the support structure surrounding the source body, made out of aluminium and stainless steel;

- the magnet boxes, that can be attached to the support structure at the sides of the source body; the magnet boxes can be filled each with up to $2 \times 4$ magnets;

- the yoke structure, made out of soft iron, for closing the magnetic field lines, consists of two bars at the side of the source and $2 \times 2$ bars on top and on bottom; the latter can be moved continuously in the horizontal direction for a closed (stronger field) or a more open configuration.

The goal of the design of this magnet frame was on the one hand to have a magnetic field map as close as possible to the magnetic field structure of the standard filter field at BATMAN which was used up to now. It was generated by $2 \times 4$ magnets embedded in the diagnostic flange (see figure 1). On the other hand it should provide a maximum in flexibility in the positioning between the diagnostic flange and the driver, in the strength and in the shape of the field.

All the magnets used both for the standard case and for the magnet frame boxes are commercially available CoSm magnets with dimensions of $9 \times 13 \times 50 \mathrm{~mm}^{3}$ with a maximum magnetic strength of $1 \mathrm{~T}$. The magnets are magnetized in the ' $13 \mathrm{~mm}$ ' direction.

However, due to the different positions of the standard magnet boxes and the magnet frame - the centre of the field in the standard case has a distance of $3 \mathrm{~cm}$ from the plasma grid, for the frame in the nearest position the distance is $9 \mathrm{~cm}$ - the standard filter field could not be matched perfectly. Instead, the magnet configuration of the frame was chosen so that in one case the maximum field strength was matched (the 'BMAX' configuration) — although in a different distance from the plasma grid-in the other case the integral $B \mathrm{~d} z$ was matched (the 'BdL' configuration) $)^{1}$.

1 The axial integral - from the plasma grid to the driver plate of the source, i.e. the exit of the driver-of the horizontal magnetic field component in the centre of the plasma source is denoted here by $B \mathrm{~d} z$, if the value is given, but also by ' $\mathrm{BdL}$ ' as the latter is the common nomenclature in the NBI community. 


\begin{tabular}{|c|c|c|c|c|c|c|c|c|}
\hline & & $\left|B_{x}\right| \max$ & $\begin{array}{l}\text { Pos. } \\
\left|B_{x}\right| \max \end{array}$ & $\begin{array}{l}\int\left|B_{x}\right| \mathrm{d} z \\
\text { source }\end{array}$ & $\begin{array}{l}\int\left|B_{x}\right| \mathrm{d} z, \\
\text { driver }\end{array}$ & $\begin{array}{l}\left|B_{x}\right| \\
\text { at PG }\end{array}$ & $\begin{array}{l}\left|B_{x}\right| \text { at } \\
\text { driver exit }\end{array}$ & $\begin{array}{l}\left|B_{x}\right| \max \text { in } \\
\text { driver }\end{array}$ \\
\hline Name & Magnets & $(\mathrm{mT})$ & $(\mathrm{cm})$ & $(\mathrm{mTm})$ & $(\mathrm{mTm})$ & $(\mathrm{mT})$ & $(\mathrm{mT})$ & $(\mathrm{mT})$ \\
\hline Standard, $z=3$ & $2 \times 4$ & 7.4 & 3.0 & 0.84 & 0.19 & 7.0 & 0.8 & 1.1 \\
\hline $\operatorname{BMAX}, z=9$ & $2 \times 4$ & 7.8 & 9.0 & 1.23 & 0.15 & 4.1 & 0.9 & 1.2 \\
\hline BMAX, $z=14$ & $2 \times 4$ & 7.8 & 14.0 & 1.33 & 0.18 & 1.4 & 3.4 & 2.8 \\
\hline $\operatorname{BMAX}, z=19$ & $2 \times 4$ & 7.8 & 19.0 & 1.10 & 0.37 & 0.2 & 6.4 & 5.9 \\
\hline $\mathrm{BdL}, z=9$ & $2 \times 3$ & 5.4 & 9.0 & 0.92 & 0.10 & 3.0 & 0.8 & 0.9 \\
\hline $\mathrm{BdL}, z=14$ & $2 \times 3$ & 5.4 & 14.0 & 0.94 & 0.13 & 1.1 & 2.5 & 2.1 \\
\hline $\mathrm{BdL}, z=19$ & $2 \times 3$ & 5.4 & 19.0 & 0.78 & 0.28 & 0.1 & 4.6 & 4.2 \\
\hline BdL, $z=9$, yoke & $2 \times 3$ & 12.5 & 9.0 & 2.48 & 0.44 & 9.5 & 5.1 & 4.6 \\
\hline
\end{tabular}

Table 1 shows the various parameters for the magnet frame configurations that have been used in the experiments so far, in comparison with the standard configuration, calculated using the 2D code QuickField [28]. The magnetic field maps for some examples are shown in figure 4, the absolute value of the horizontal magnetic field strength in the centre of the ion source as a function of the distance from the plasma grid is shown in figure 5. Almost all fields show a field-free region in the source or in the driver; this region is moved deep into the driver, when moving the filter field towards the driver exit.

Two different numbers of magnets $(2 \times 4,2 \times 3)$ at three different positions $(z=9$, i.e. close to the plasma grid, $z=14$, i.e. source centre, and $z=19$, i.e. close to the driver) have been used for the results reported in this paper. Additionally, the magnet boxes have been exchanged so that the horizontal field direction changed by $180^{\circ}$ in order to change the drift of the plasma and hence the top/down asymmetry. This might be important as the IPP prototype source has an intrinsic asymmetry due to the position of the Cs oven (top of the source, see figure 2). Also some few experiments have been performed without any field and with the yoke closed in the 'BdL, $z=9$ ' configuration.

With the present setup, the various parameters of the magnetic field configuration are not completely independent from each other. This is shown in figure 6, where the correlation of the magnetic field at the driver exit and the integral $B \mathrm{~d} z$ value with the magnetic field at the plasma grid is shown. These parameters are chosen as the performance of the source is correlated with them (see below). However, it can be seen that the magnetic field at the plasma grid can be varied in a wide range without changing the integral $B \mathrm{~d} z$ considerably and that there is only some correlation of the field at the driver exit with the field at the plasma grid for low fields.

\section{Results and discussion}

\subsection{Source performance}

Figure 7 shows the comparison of the overall performance of the ion source with respect to the maximum achieved extracted current density and the minimum number of co-extracted electrons. Also the comparison with the record values for hydrogen obtained in 2005 with the standard magnetic field is shown [5]. This comparison, however, suffers from problems with the measurement of the RF power at BATMAN in 2005; after installing a new RF power 

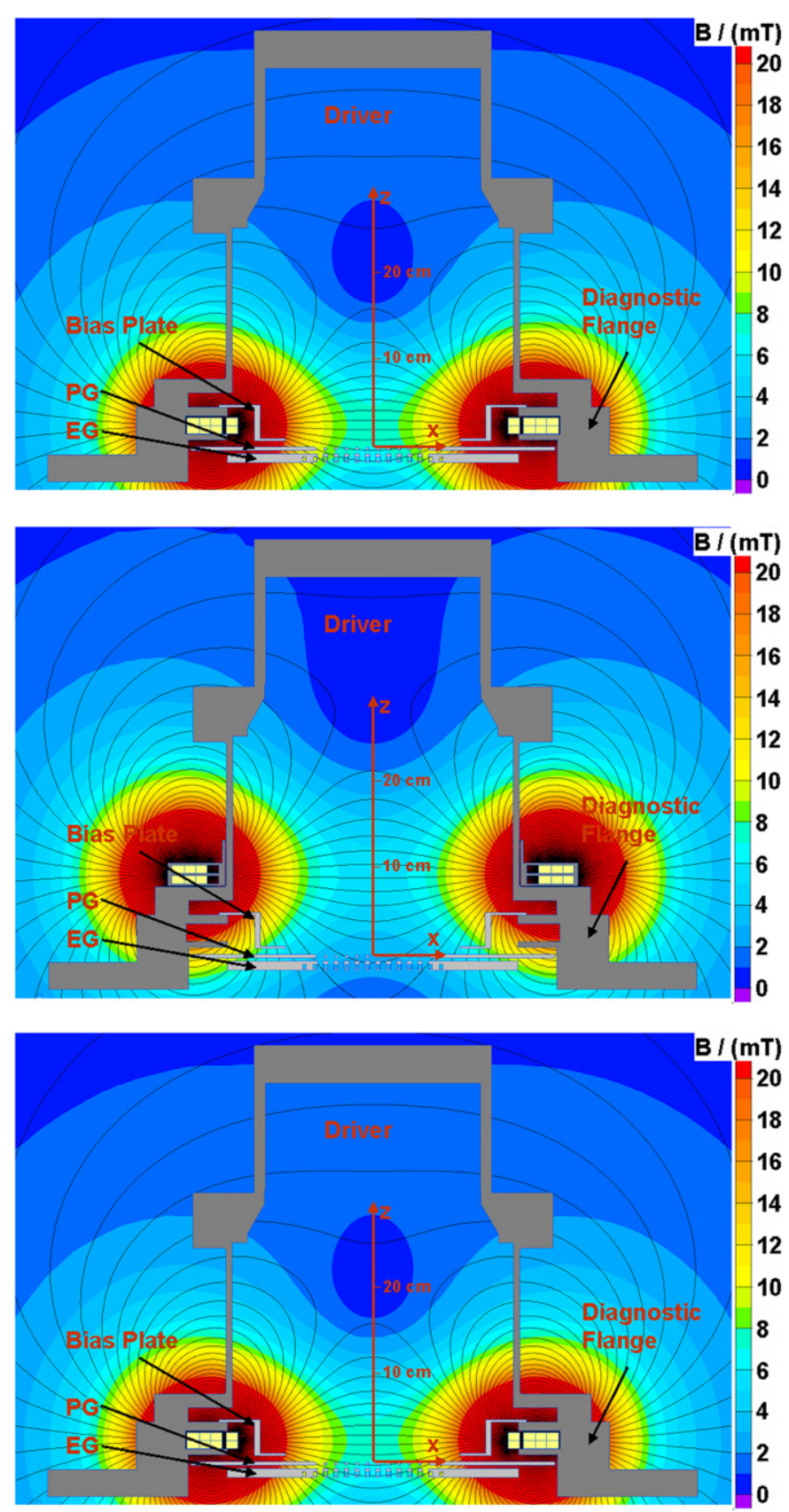

Figure 4. Some 2D maps of the horizontal magnetic field $\left(B_{x}\right)$ component for (top) the standard filter field with the magnets embedded in the diagnostic flange; (middle) for the 'BdL, $z=9$ ' case and (bottom) for the 'BdL, $z=14$ ' case.

measurement system in 2007 it turned out that the power levels measured before should be reduced by typically $30 \%$. This was done in this paper for the RF power values for the standard field, but there is still an uncertainty as the reduction factor is not fixed but varies in a certain range depending on the matching and other parameters. 


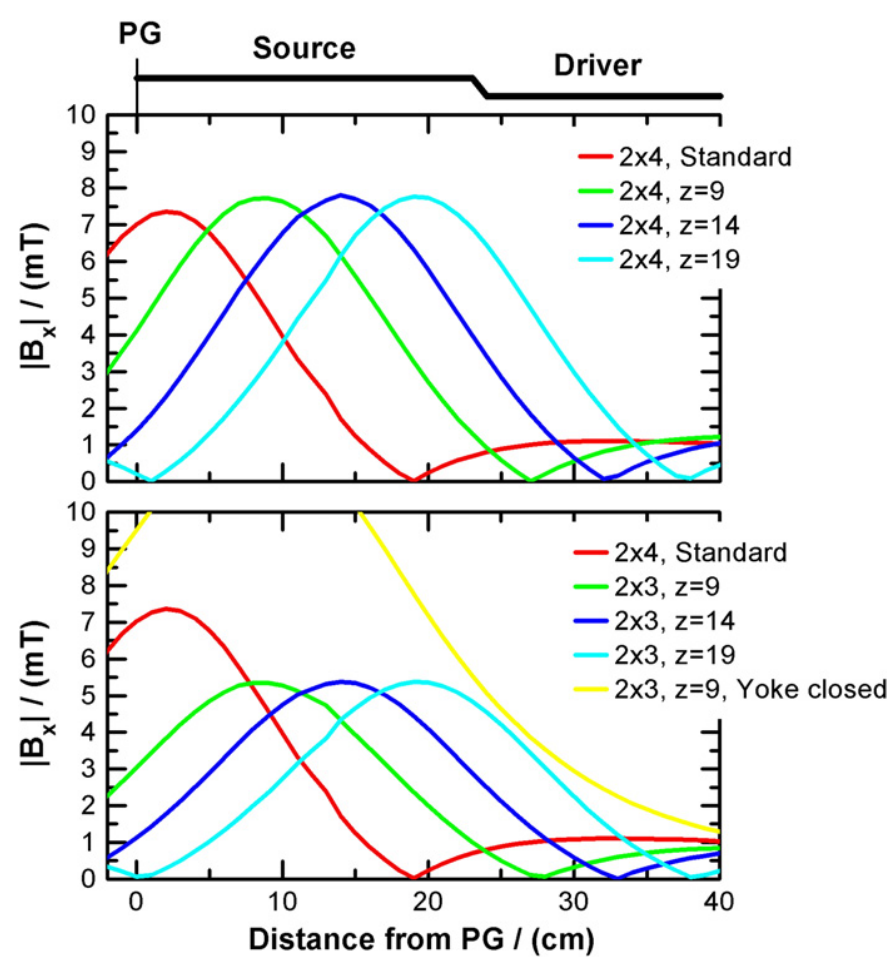

Figure 5. Horizontal (' $B_{x}$ ') magnetic field strength in the centre of the ion source in dependence of the distance from the plasma grid ( ' $z$ ' direction) for the configurations used in the experiments. Top: standard and the respective 'BMAX' matched configurations $(2 \times 4)$. Bottom: standard and the respective 'BdL' matched configurations $(2 \times 3)$ together with a configuration with the yoke closed.

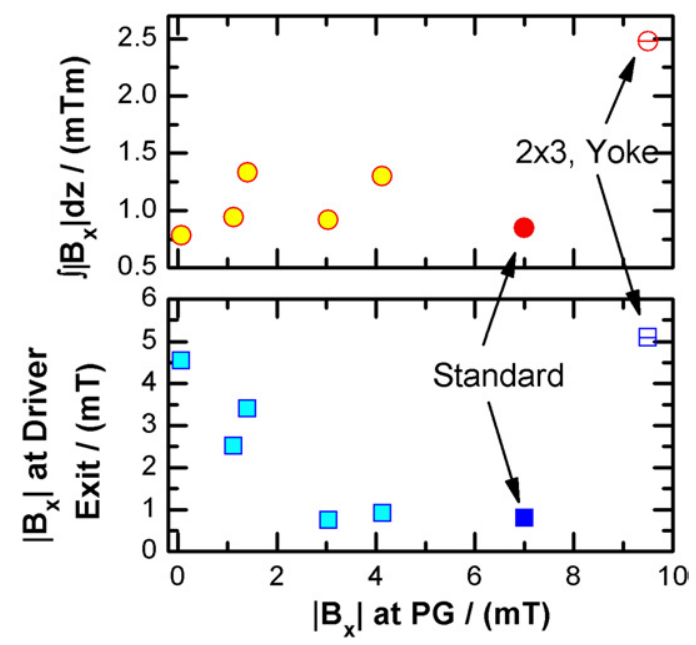

Figure 6. Correlation of the magnetic field strength at the exit of the driver and the integral $B_{x} \mathrm{~d} z$ value with the magnetic field strength at the plasma grid for the used magnetic field configurations (see table 1). 


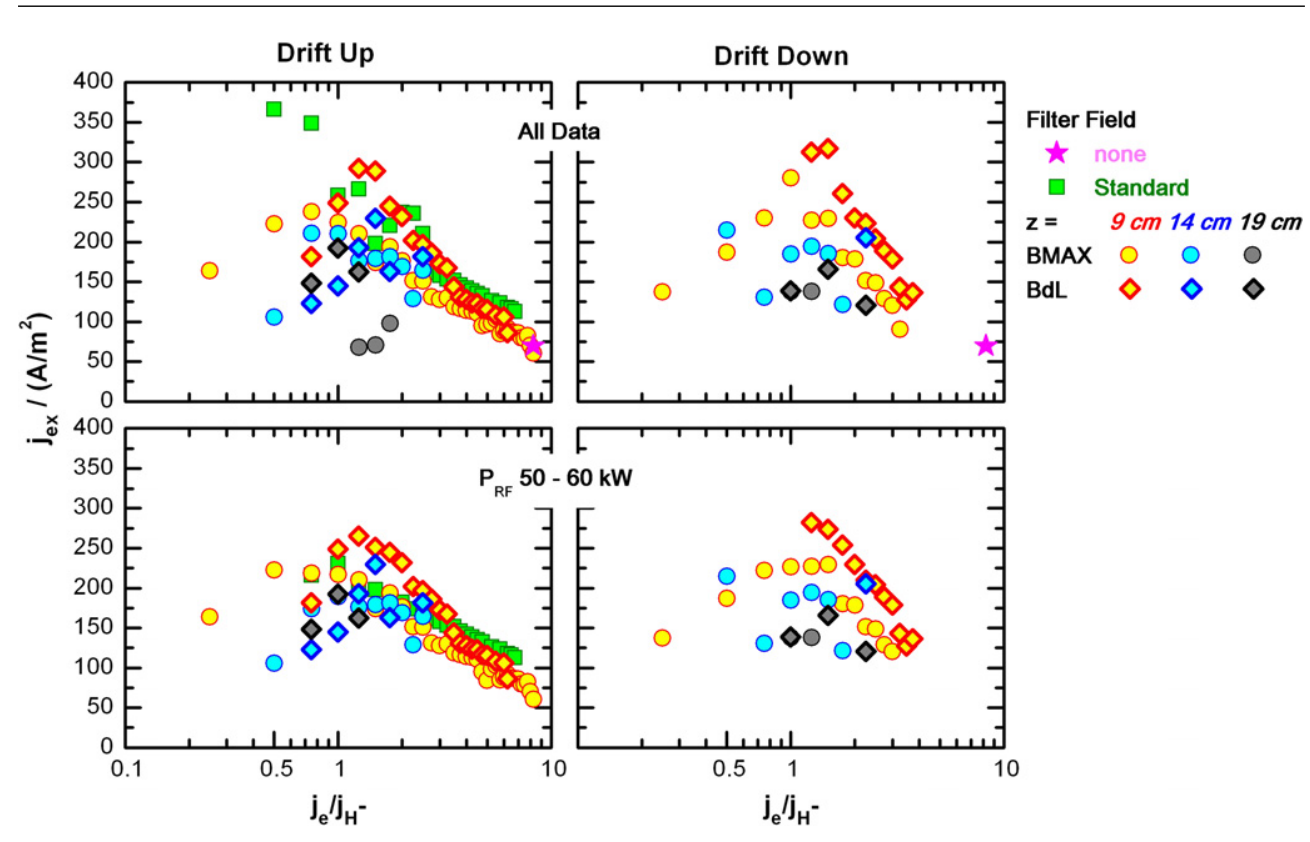

Figure 7. Performance of the IPP prototype source for hydrogen operation in dependence of the magnetic field configuration. Shown are the maximum achieved values of the extracted current density for intervals of 0.25 of the ratio of the co-extracted electron current to the negative ion current (the number refers to the upper bound). Left column: filter field in the drift-up configuration, right column: filter field in the drift-down configuration. Top row: complete data set; bottom row: RF power between 50 and $60 \mathrm{~kW}$.

Nevertheless, the data in figure 7 show that

- the maximum current density is achieved for the flexible magnetic setup cases with the 'BdL' field configuration, although the electron ion ratio is still just above 1;

- there is not much difference in the source performance between the standard case and the 'BMAX, $z=9$ ' configuration near the plasma grid, if same parameters, i.e. RF power, are compared;

- the performance does not depend much on the drift direction;

- the performance decreases when the field is shifted towards the driver.

The overall performance with the standard field configuration, i.e. ion current densities well above $300 \mathrm{~A} \mathrm{~m}^{-2}$ with an electron/ion ratio well below 1, however, could not be achieved with the flexible magnet frame configurations. Especially, the negative ion current density decreases with decreasing electron/ion ratio for values less than 1. Also there might be a principle restriction in source performance; this behaviour might also be a consequence of the course of the experiments: The source was not 'forced' to optimum performance, i.e. increasing RF power, lengthy Cs conditioning, etc for each of the magnetic configuration.

Most of the data have been achieved at a source filling pressure below $0.4 \mathrm{~Pa}$. In this pressure range, however, it was difficult to sustain the plasma in the driver, when the filter field position was near the driver exit. In most cases, the plasma went out after the initial gas puff which is necessary for plasma ignition, when the pressure in the driver got very low. A possible explanation is the neutral depletion, i.e. the depletion of neutral gas, the latter being necessary to couple the RF power in the driver [29]. If this is the case, the observed behaviour 


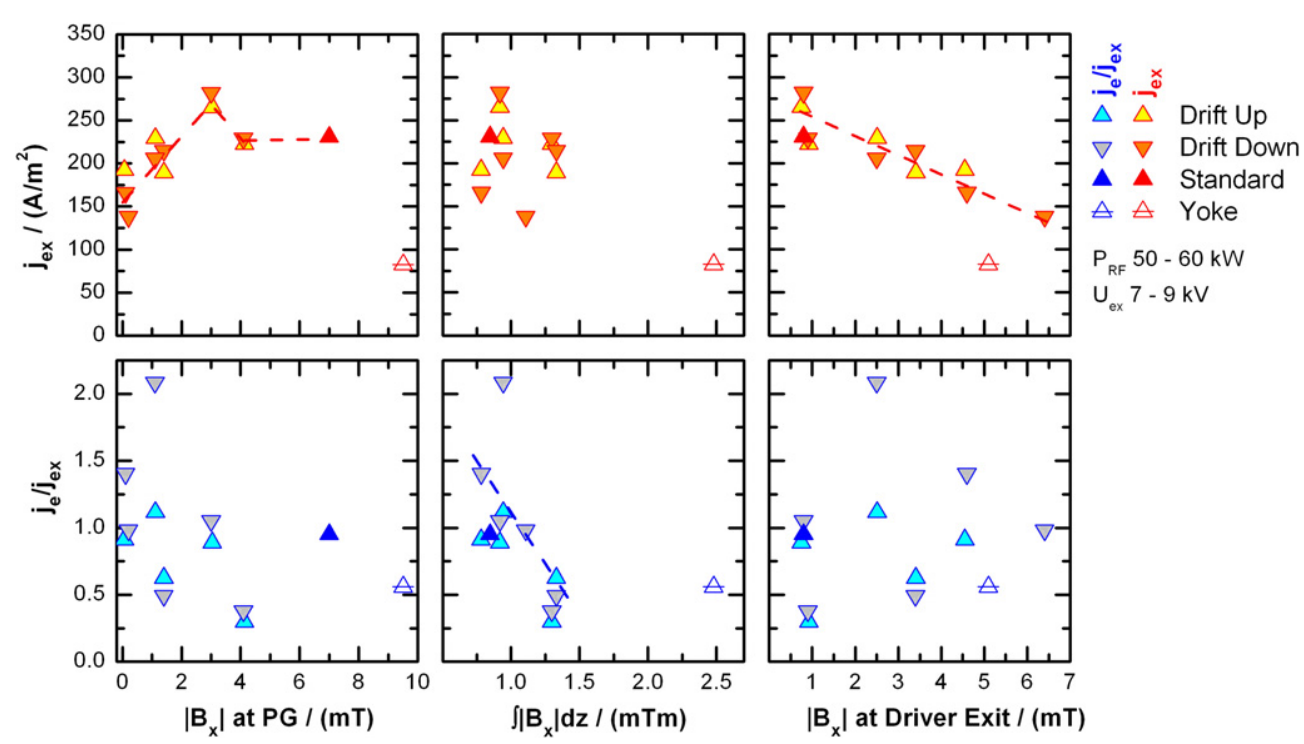

Figure 8. Performance of the IPP prototype source for hydrogen operation in dependence of the magnetic field parameters. Shown are the maximum achieved values of the extracted current density and the minimum achieved values of the ratio of the co-extracted electron current to the negative ion current. Left: in dependence on the magnetic field strength at the plasma grid. Centre: in dependence on the integral $B \mathrm{~d} z$, value. Right: in dependence on the magnetic field strength at the driver exit. Data sets are chosen for an RF power between 50 and $60 \mathrm{~kW}$ and an extraction voltage of 7 to $9 \mathrm{kV}$.

indicates that the neutral depletion seems to be enhanced when there is a field in the driver. The latter, however, is also the case for the large RF sources where the filter field is generated by a current through the plasma grid. Hence, the sustainment of the driver plasma with magnetic field inside the driver at low source pressure is an open issue. This, however, seems to be a problem especially for the small IPP prototype source; low pressure operation down to $0.2 \mathrm{~Pa}$ was possible in the large 1/2-size ITER source $(1 \mathrm{~m} \times 1 \mathrm{~m})$ at the IPP RADI test facility even with a magnetic field strength comparable to the values here [30]. A similar dependence of the operating pressure from the volume to surface area ratio was seen for the arc driven sources [10].

Figure 8 shows the maximum achieved extracted current density and the minimum achieved ratio of co-extracted electron to ion current in dependence on some parameters of the magnetic field configurations, i.e. on the magnetic field strength at the plasma grid and at the driver exit as well as on the integral $B_{x} \mathrm{~d} z$ in the source. The data sets are independent, i.e. represent not necessarily the same discharge.

The figure clearly shows that the extracted ion current density correlates with the magnetic field at the plasma grid and at the driver exit (both quantities have, however, some correlation, see figure 6), whereas the number of co-extracted electrons correlates with the integral $B_{x} \mathrm{~d} z$ in the ion source. The maximum current density was achieved if the magnetic field strength at the plasma grid is around $3 \mathrm{mT}$ and the magnetic field strength at the driver exit is low; the number of co-extracted electrons is at lowest if the integral $B_{x} \mathrm{~d} z$ value is around $1.25 \mathrm{mTm}$. However, these magnetic field conditions could not be achieved simultaneously with the present technical possibilities. The data also show again that there is not much difference of the source performance if the drift of the plasma is downwards or upwards. 


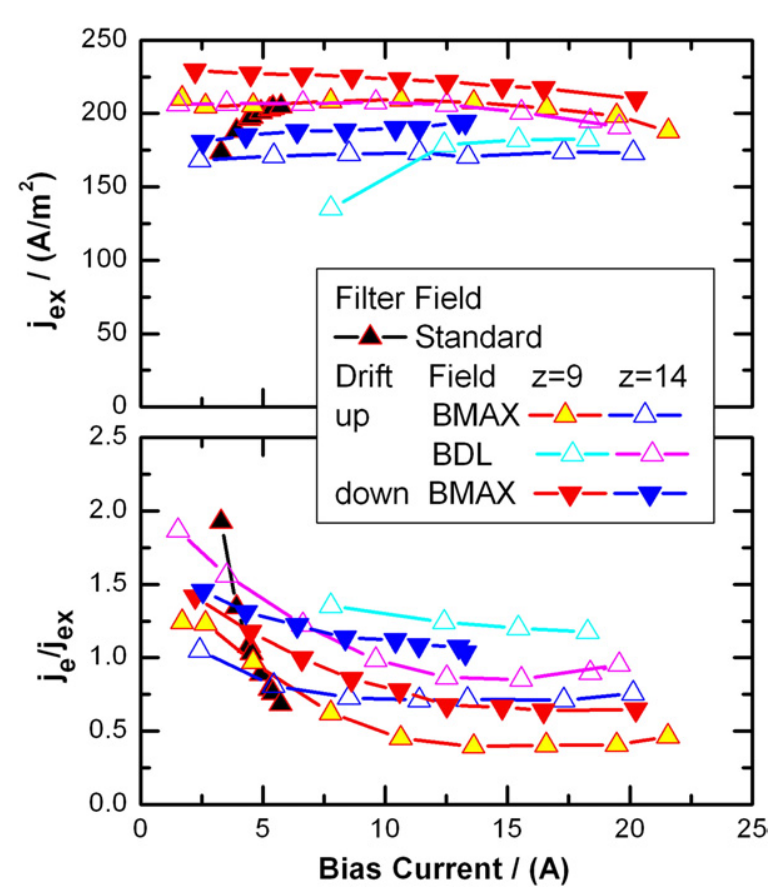

Figure 9. Performance of the IPP prototype source in dependence of the bias current for the different magnetic field configurations. The data for the 'BMAX' and 'BdL' configurations have been obtained in dedicated bias scans; the data for the standard configuration have been obtained during conditioning the source. All data are for $50-55 \mathrm{~kW}$ RF power and $9 \mathrm{kV}$ extraction voltage.

The observed dependence of the extracted negative ion and electron currents on the magnetic field configuration cannot be explained by a simple model, as there is a complex interplay between the different fluxes of positive ions- which are needed for the space charge compensation of the negative ions - and electrons from the driver to the plasma grid and the negative ion flux coming from the plasma grid into the boundary layer. For example, ion transport calculations [16] with the IPP Trajan code showed that the extraction probability of negative ions increases monotonically with increasing filter field strength. This is in some contradiction to the findings here. But both results from experiments and model fit into the overall picture: as the production and transport of the negative hydrogen ions occur in the boundary layer near the plasma grid, the magnetic field near the grid plays an important role; the electrons, in contrast, are generated in the driver, so that their transport is determined by the magnetic field in the expansion region, represented by the integral $B_{x} \mathrm{~d} z$.

A remarkable difference between the standard magnetic case and the magnet frame configurations is the much larger bias current needed for optimum electron suppression, 2-5 A in the standard magnetic field configurations (see also [4,8]), and 10-12 A for the magnet frame configurations. This is shown in figure 9. For comparison, also data for the standard configuration are shown, although some care should be taken in the comparison due to the problems of the RF power measurements discussed above, and due to the fact that these data have not been obtained during a dedicated bias scan experiment as the data for the configurations with the magnet frame. In all cases with the magnet frame configuration, the extracted ion current density depends only weakly on the bias current, whereas the dependence on the magnetic field is much more pronounced. However, the number of co-extracted electrons does also depend on the bias current, reaching some saturation value in the range 10-12 A. 


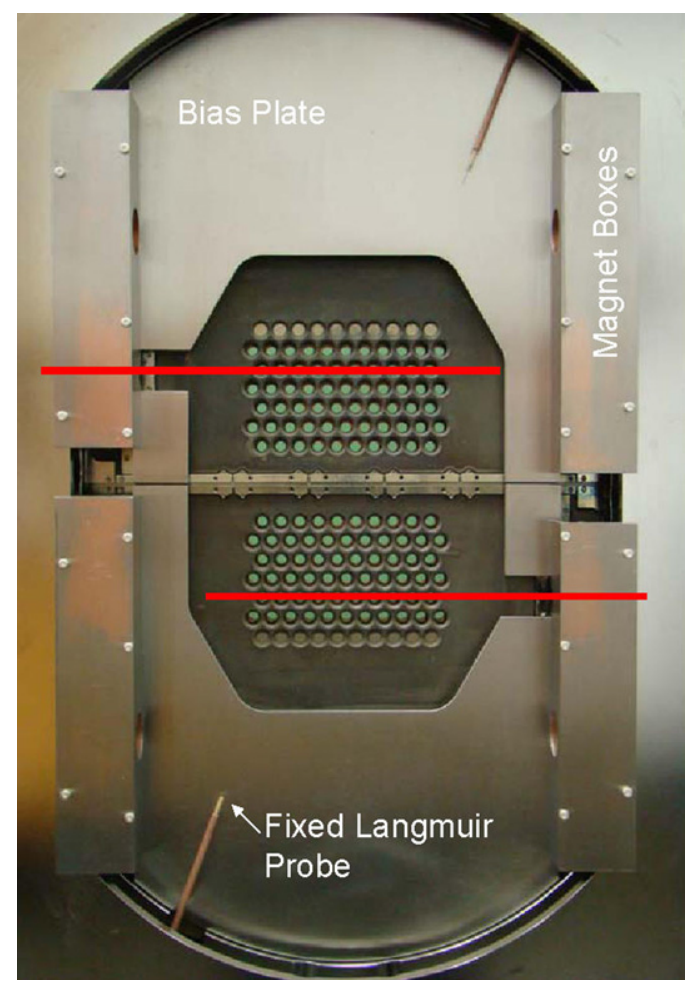

Figure 10. View of the plasma grid with bias plate of the IPP prototype source at BATMAN. Also indicated are the fixed Langmuir probes and the line-of-sights for the OES measurements of the plasma asymmetry. Both line-of-sights have a distance of $7 \mathrm{~mm}$ from the plasma grid and an optical diameter of about $10 \mathrm{~mm}$.

Whether this discrepancy in the plasma grid bias behaviour is related to the larger distance of the maximum field strength from the plasma grid is still an open issue. It is, however, accompanied by changes in the plasma parameters: Langmuir probe measurements show a much larger plasma potential for the configurations with the magnet frame than for the standard configuration [21].

\subsection{Source symmetry}

The plasma asymmetry $S_{\mathrm{p}}$ in the negative ion source is defined in this report by

$$
S_{\mathrm{p}}=\frac{\int \varepsilon_{\mathrm{H}_{\beta}} \mathrm{d} x_{\text {top }}}{\int \varepsilon_{\mathrm{H}_{\beta}} \mathrm{d} x_{\text {bottom }}}-1 ;
$$

the corresponding horizontal (' $x$ ') line-of-sights are shown in figure 10; the used line-of-sights collect the light nearly in the centre of both grid halves at a distance of $7 \mathrm{~mm}$ from the plasma grid with an optical diameter of about $10 \mathrm{~mm}$. The $\mathrm{H}_{\beta}$ line emission is used, as it is in the most cases available, the $\mathrm{H}_{\alpha}$ line is frequently overexposed when the spectrometers are used for measurements of the Cs $852 \mathrm{~nm}$ line. Furthermore, the $\mathrm{H}_{\beta}$ emission is solely caused by collision of atoms and ions with electrons and hence reflects the 'plasma' conditions, whereas the $\mathrm{H}_{\alpha}$ emission also results from mutual neutralization of positive and negative ions [6].

With this definition, a homogeneous source is characterized by $S_{\mathrm{p}}=0$. Positive values of $S_{\mathrm{p}}$ indicate that the plasma Balmer emission is more pronounced at the upper 


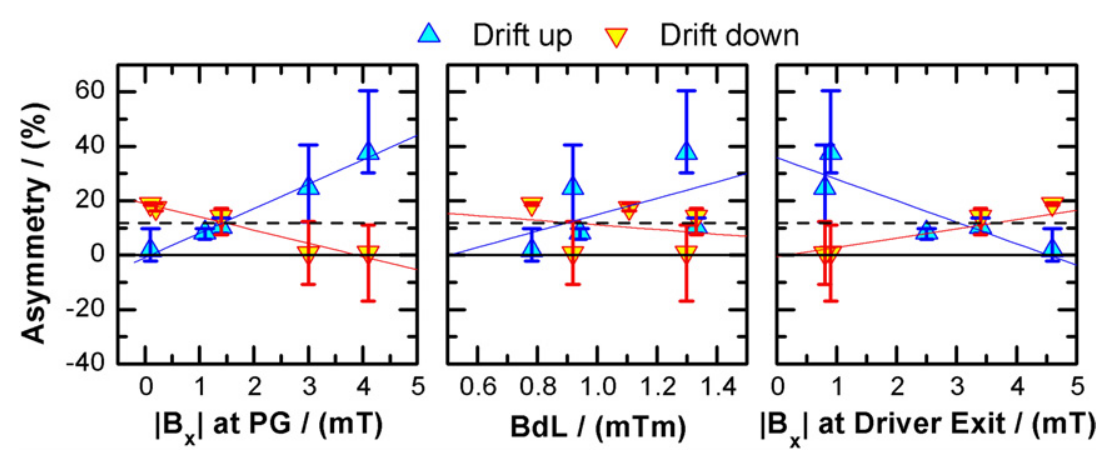

Figure 11. Dependence of the plasma asymmetry near the plasma grid on the magnetic field parameters. Shown are the average values as well as the maximum and the minimum values, indicated by the bars, for pulses with an RF power between 50 and $60 \mathrm{~kW}$, an extraction voltage between 7 and $9 \mathrm{kV}$ and a bias current between 9 and $12 \mathrm{~A}$.

part of the source, i.e. a drift upwards, negative values of $S$ indicate accordingly a drift downwards.

Figure 11 shows the dependence of the plasma asymmetry on some of the magnetic field parameters for the cases with the magnet frame. The bars do not indicate the error, but the upper and lower values of the respective data set and so the range of the data for a given magnetic field configuration. There is a strong linear correlation between the plasma asymmetry and the magnetic field strength at the plasma grid, less correlation with the magnetic field strength at the driver exit - the latter two magnetic field quantities have also some correlation in this range (see figure 6) — and no correlation at all between the plasma asymmetry near the plasma grid and the integral magnetic field $B_{x} \mathrm{~d} z$ in the source. This indicates that also the plasma drift near the plasma grid, not only the negative ion transport, is determined by the magnetic field near the plasma grid.

Furthermore, the dependence of $S_{\mathrm{p}}$ on the magnetic field strength is reversed when the magnetic field direction, i.e. the plasma drift direction, is reversed. However, this dependence is not symmetrical with respect to zero, it seems that there is an 'intrinsic' offset of about $12 \%$ plasma asymmetry.

Although this 'intrinsic' offset of the plasma asymmetry might be caused by measurements errors - the error of the $\mathrm{H}_{\beta}$ line emission is in the range of $10 \%$, it might also be caused by the asymmetric position of the Cs oven. The plasma asymmetry in the IPP cesiated negative hydrogen ion source has in principle two causes: (1) the vertical plasma drift in the filter field leading to an asymmetry of the transport of the negative ions-due to an asymmetric distribution of positive ions needed for space charge compensation - and of the destruction of negative ions by electron collisions; (2) an asymmetry of the negative ion production at the plasma grid due an asymmetric quality of the Cs layer at the plasma grid as the Cs oven is positioned on top of the source.

\section{Conclusions}

The dependence of the performance of the IPP RF driven prototype source for negative hydrogen ions on the magnetic filter field was investigated with a new flexible magnetic setup at the IPP test facility BATMAN.

Two clear correlations of the source performance with magnetic field parameters have been found. For ion extraction as well as for the plasma asymmetry, the magnetic field strength 
in front of the plasma grid is the important parameter, whereas the number of co-extracted electrons is determined by the integral BdL from the driver to the plasma grid. For hydrogen, a field of about $3 \mathrm{mT}$ in front of the plasma grid for sufficient negative hydrogen ion extraction is needed, for sufficient electron suppression an integral BdL of more than $1.2 \mathrm{mTm}$ is needed. Both dependences are consistent with the present understanding of the processes in an RF driven negative ion source and are related to the different creation locations of negative ionsat the plasma grid-and electrons — in the driver.

The results are an important input for the design of the magnetic filter field of the large ITER NBI source which is currently under way.

\section{Acknowledgments}

The work was supported by a grant from F4E (F4E-2008-GRT-007) and by an R\&D contract from ITER IO (ITER_CT_09_4100000772_0609). The authors are solely responsible for the content.

\section{References}

[1] Hemsworth R et al 2008 Rev. Sci. Instrum. $7902 \mathrm{C} 109$

[2] Schunke B et al 2008 Proc. 1st Conf. on Negative Ions, Beams and Sources (Aix-en-Provence, France, 10-12 September 2008) AIP Conf. Proc. 1097480

[3] Hemsworth R et al 2009 Nucl. Fusion 49045006

[4] Speth E et al 2006 Nucl. Fusion 46 S220

[5] Franzen P et al 2007 Nucl. Fusion 47264

[6] Fantz U et al 2007 Plasma Phys. Control. Fusion 49 B563-80

[7] Stäbler A et al 2009 Proc. 25th Symp. on Fusion Technology (Rostock, Germany, 15-19 September 2008) Fusion Eng. Des. 84265

[8] Franzen P et al 2008 Proc. 1st Conf. on Negative Ions, Beams and Sources, AIP Conf. Proc. 109745

[9] Tsumori K et al 1995 Fusion Eng. Des. 26 473-83

[10] Takeiri Y et al 2010 Rev. Sci. Instrum. 81 02B114

[11] Tobai Het al 2008 Rev. Sci. Instrum. $7902 \mathrm{c} 111$

[12] Hanada M et al 2006 Rev. Sci. Instrum. 77 03A515

[13] Hanada M et al 2005 Fusion Eng. Des. 74 311-7

[14] Takeiri Y et al 2007 AIP Conf. Proc. 925211

[15] Fantz U et al 2009 Nucl. Fusion 49125007

[16] Gutser R et al 2009 Plasma Phys. Control. Fusion 51045005

[17] Holmes A J T 1982 Rev. Sci. Instrum. 531523

[18] Hatayama A et al 2006 Rev. Sci. Instrum. 77 03A530

[19] Taccogna F 2010 Phys. Plasmas 17063502

[20] Lishev S et al 2010 J. Phys.: Conf. Ser. 223012003

[21] Schiesko L, McNeely P, Fantz U, Franzen P and NNBI Team 2011 Plasma Phys. Control. Fusion 53085029

[22] Dietrich S et al 2007 Proc. 27th ICPIG Conf. Prague (Czech Republic, 15-20 July 2007) 4P06-37, p 1657

[23] Takado N et al 2006 Rev. Sci. Instrum. 77 03A533

[24] Franzen P et al 2010 Proc. 2nd Conf. on Negative Ions, Beams and Sources (Takayama, Japan, 16-19 November 2010) AIP Conf. Proc. $1390310-21$

[25] Heinemann B et al 2009 Proc. 25th Symp. on Fusion Technology (Rostock, Germany, 15-19 September 2008) Fusion Eng. Des. 84915

[26] Marcuzzi D et al 2009 Proc. 25th Symp. on Fusion Technology (Rostock, Germany, 15-19 September 2008) Fusion Eng. Des. 841253

[27] Heinemann B et al 1998 Proc. 20th Symp. on Fusion Technology, (Marseille, France, 1998) Association EURATOM-CEA, Saint Paul Lez Durance p 4331

[28] http://www.quickfield.com

[29] McNeely P, Wünderlich D and the NNBI Team 2011 Plasma Sources Sci. Technol. 20045005

[30] Franzen P 2011 Continuation of the development of the RF ion source 2010 Technical Report Max-Planck-Institut für Plasmaphysik, February 2011, IPP 4-290 